\title{
The Stochastic Knapsack Revisited: Switch-Over Policies and Dynamic Pricing
}

\author{
Grace Y. Lin, Yingdong Lu \\ IBM T.J. Watson Research Center \\ Yorktown Heights, NY 10598 \\ E-mail: \{gracelin, yingdong\}@us.ibm.com \\ David D. Yao* \\ IEOR Dept., Research \\ Columbia University \\ New York, NY 10027 \\ E-mail: yao@ieor.columbia.edu
}

(revision: April 2006)

\begin{abstract}
The stochastic knapsack has been used as a model in wide ranging applications from dynamic resource allocation to admission control in telecommunication. In recent years, a variation of the model has become a basic tool in studying problems that arise in revenue management and dynamic/flexible pricing; and it is in this context that our study is undertaken. Based on a dynamic programming formulation and associated properties of the value function, we study in this paper a class of control that we call switch-over policies start from accepting only orders of the highest price, and switch to including lower prices as time goes by, with the switch-over times optimally decided via convex programming. We establish the asymptotic optimality of the switch-over policy, and develop pricing models based on this policy to optimize the price reductions over the decision horizon.
\end{abstract}

\section{Introduction}

The stochastic knapsack refers in general to a dynamic resource allocation problem in which a fixed amount of resource is allocated sequentially to random demands of multiple classes. The problem appears to have many different roots. In Derman et al (1972), a Markov decision problem is formulated to determine how to assign fixed amount of different resources to sequential arrivals with random unit returns. In Prastacos (1983), a problem for sequential investment is analyzed which allows only one acceptance decision to be made over the horizon.

${ }^{*}$ Research undertaken while an academic visitor at IBM T.J. Watson Research Center; also supported in part by NSF grant DMI-0085124 and Hong Kong RGC Grant CUHK4173/03E. 
In the late 1980's, the stochastic knapsack was used as a model to study admission control in telecommunication networks, so-called loss networks in particular; refer to Ross and Tsang [16], and Ross and Yao [17].

In recent years, one version of the stochastic knapsack has become a basic model in studying problems in the general area of revenue management. Here the capacity of the knapsack corresponds to a given amount of resource that can be used to fulfill customer demand, over a given time frame that is typically quite short. Some examples include: rooms in a hotel targeted for weekend tourists, seats on an airplane that must be sold before departure, fashion items at a department store designed for a particular season. Refer to Kleywegt and Papastavrou [10, 11, Papastavrou et al. [15], and Van Slyke and Young [20].

Our study here also falls into this category, although it is motivated by a new application. A major producer of personal computers (PC's) from time to time has to liquidate a substantial inventory of desktop or laptop computers returned from corporate leases. This involves a number of sales channels - including catalog direct sales, dealers/brokers, and on-line auction - which differ in both price and batch size. What is needed by practitioners involved in this process is a tool to support pricing decisions. Specifically, not only what type of "bids" (customer demand or offer to buy the PC's) to accept and when (in terms of time and available inventory), but also how to price the sales over time. Refer to more details in [4, 5].

As evident from previous studies, with prices given, the problem of when to accept or reject which demand can be formulated and solved using dynamic programming (DP). This, however, does not in general lead to optimal policies that have simple and easy-to-implement structures; neither does it result in a tractable value function, upon which pricing optimization can be carried out. Our approach makes use of certain properties of the DP value function, such as concavity and submodularity (which have been familiar properties in other application contexts such as queueing control, e.g., Lippman [13]), which lead to a lower- and upper-orthant structure of the optimal policy (see Proposition 1). This structure, in turn, motivates us to focus on a class of "switch-over" policies.

The switch-over policy is executed in the following manner: it starts from accepting only orders of the highest price, and gradually switches to including lower prices as time goes by. There are several advantages in focusing on this class of policies: a) they are consistent with the lower- and upper-orthant structure associated with the optimal policy; b) they are easy to identify: the optimal switch-over times are readily derived through convex programming; and most importantly, c) they are practical for implementation: indeed they follow closely the prevailing practice in certain industry sectors. Furthermore, we can prove that the switch-over policy, while sub-optimal in general, is asymptotically optimal in the sense that the relative 
error between the switch-over and the optimal policies goes to zero as the available inventory increases to infinity (along with the planning horizon).

One aspect of our work that is a departure from previous studies is that we are concerned with setting the optimal prices (in addition to deciding order acceptance/rejection). Based on the switch-over policy, we formulate optimization problems, so that the reduced ("sales") prices over the decision horizon can be optimally determined, taking into account that the rate of demand is a (decreasing) function of the price. A closely related set of papers in revenue management, although not always making an explicit connection to the stochastic knapsack, studies problems that are similar to ours in both physical and mathematical aspects. These include Bitran and Mondschein [1], Brummelle and Walczak [2, Feng and Gallego [6, 7], Feng and Xiao [8], Lee and Hersh [12], and Zhao and Zheng [21].

Briefly, the rest of the paper is organized as follows. In 92 we start with a dynamic programming problem formulation, and bring out the structure of the optimal policy. In the next two sections, we focus on the switch-over policy, starting with the case of constant batch sizes in 93 . The general case of random batch sizes is studied in 94 , and the asymptotic optimality of the switch-over policy is established in 95 . We then develop the pricing models in \$6, and conclude with possible extensions in 97 .

\section{The Dynamic Programming Formulation}

Here is a formal description of our model. There are $W$ units of inventory available to supply the orders at times $n=1, \cdots, T$, where $T$ is a given integer, representing the planning horizon. The order (demand) that arrives in each period $n$ takes the form of a bivariate random vector: $\left(P_{n}, Q_{n}\right)$, where the two components represent the unit offer price and the required quantity. Suppose $\left(P_{n}, Q_{n}\right)$ are i.i.d. across $n$, following a joint distribution:

$$
\mathrm{P}\left[P_{n}=p_{i}, Q_{n}=j\right]:=\theta_{i j}, \quad i=1, \ldots, m ; \quad j=1, \ldots, W .
$$

Here, we assume, for all $i$ and $j, p_{i}>0$ and $\sum_{i} \sum_{j} \theta_{i j} \leq 1$, with

$$
\theta_{0}:=1-\sum_{i} \sum_{j} \theta_{i j} \geq 0
$$

representing the probability that there is no order arrival in a period.

In each period, our decision is, after observing the realized $\left(P_{n}, Q_{n}\right)$, whether or not to supply the order. If we do, a revenue of $P_{n} Q_{n}$ is collected; otherwise, we earn nothing. Here, we assume that each order is either supplied in full, or not at all; i.e., no partial supply is allowed. In particular, if the inventory available upon an order arrival is less than the order 
size, then no supply takes place. The objective is to maximize the expected revenue collected over the planning horizon of $T$ periods and the total available inventory of $W$ units.

Let $V(n, d)$ denote the expected revenue we can collect, under optimal actions, starting from period $n(\leq T)$, with $d(\leq W)$ units of inventory left. Then, we have the following dynamic programming (DP) recursion:

$$
\begin{aligned}
V(n, d) & =V(n+1, d)\left[\theta_{0}+\Theta(d)\right] \\
& +\sum_{i} \sum_{j \leq d} \theta_{i j} \cdot \max \left\{p_{i} j+V(n+1, d-j), V(n+1, d)\right\},
\end{aligned}
$$

where

$$
\Theta(d):=\sum_{i} \sum_{j>d} \theta_{i j}
$$

Clearly, the first term on the right hand side of (2) corresponds to the case of either no arrival or the order size exceeds the available inventory; whereas each term under the double summation compares the two actions: accept (i.e., supply) the order, or reject it. If we supply the order, then we earn the revenue $p_{j} j$, and proceed to the next period with $j$ units less in the available inventory. In the last period, we have

$$
V(T, d)=\sum_{i} \sum_{j \leq d} \theta_{i j} p_{i} j
$$

since clearly the best action is to supply any possible order using all the remaining inventory.

The above DP is quite easy to solve - the overall computational effort is, after all, only $O(T W)$. Short of any structural properties, however, the solution does not readily translate into a policy that is easy to implement. We need to pre-compute and store the $V(n, d)$ values for all $n=1, \ldots, T$ and all $d=1, \ldots, W$. Then, after observing the realized demand $\left(P_{n}, Q_{n}\right)$, we will supply it, if $Q_{n} \leq d$ and

$$
P_{n} Q_{n}+V\left(n+1, d-Q_{n}\right) \geq V(n+1, d)
$$

and reject it otherwise. Furthermore, the lack of a tractable form of the value function with respect to the prices is a severe handicap when it comes to solving the optimal pricing problem.

In the case that all orders are of unit size, the above problem can be reduced to a special case of a certain queueing control model in Lippman [13], and the value function, $V(n, d)$, can be shown to be concave in $d$ and submodular in $(n, d)$. These properties have the following implications:

- For each price type $i$, if the order is rejected in some state $\left(n^{*}, d^{*}\right)$, then it is rejected in all "lower" states $(n, d) \leq\left(n^{*}, d^{*}\right)$; if the order is accepted in some state $\left(n^{*}, d^{*}\right)$, then it is accepted in all "upper" states $(n, d) \geq\left(n^{*}, d^{*}\right)$. 
To understand the above, we know from the DP recursion, if a type $i$ order is rejected in state $\left(n^{*}, d^{*}\right)$, then

$$
p_{i} \leq V\left(n^{*}, d^{*}\right)-V\left(n^{*}, d^{*}-1\right) .
$$

For any state $(n, d) \leq\left(n^{*}, d^{*}\right)$, we have

$$
V\left(n^{*}, d^{*}\right)-V\left(n^{*}, d^{*}-1\right) \leq V\left(n^{*}, d\right)-V\left(n^{*}, d-1\right) \leq V(n, d)-V(n, d-1),
$$

due to the concavity (the first inequality) and submodularity (the second inequality) of $V$. Hence,

$$
p_{i} \leq V(n, d)-V(n, d-1)
$$

i.e., the same order should also be rejected in state $(n, d)$ as well, which is the lower-orthant property. On the other hand, if the order is accepted in state $\left(n^{*}, d^{*}\right)$, then it must be accepted in any state $(n, d) \geq\left(n^{*}, d^{*}\right)$; for if it is rejected in state $(n, d)$, then following the lower-orthant property, it must also be rejected in state $\left(n^{*}, d^{*}\right)$.

The above properties, stated in a slightly different but equivalent form below, are the basis for the switch-over policies that we shall focus on in the rest of this paper.

Proposition 1 Suppose all orders are of the same, constant size. (Hence, without loss of generality, assume this constant size is unity, i.e., $Q_{n} \equiv 1$.) Then, the optimal policy has the following structure: for each inventory level $d$, there exist time epochs $0=t_{0}(d) \leq t_{1}(d) \leq$ $\cdots \leq t_{m}(d)=T$ such that a price type $k$ order is accepted (rejected) if and only if $n \geq t_{k-1}(d)$ $\left(n<t_{k-1}(d)\right)$; and for each $k, t_{k}(d)$ is decreasing in $d$.

Throughout the paper, we use "increasing" and "decreasing" in the non-strict sense.

\section{The Switch-Over Policy}

The structural result in Proposition 1 suggests a threshold type policy as follows. For each type of order $i$, there is a critical state $\left(n_{i}, d_{i}\right)$ - supply the order if and only when time has reached $n_{i}$ or beyond and inventory is at least $d_{i}$. This policy, however, is still difficult to analyze (in terms of deriving an explicit objective function to be used for pricing optimization). What we do below is to reduce this two-dimensional threshold policy to a single dimension, in time only. Specifically, we start with accepting only orders with the highest price, until a time $t_{1}$, when we start to accept orders of the top two prices, until a time $t_{2}$, when we start to accept orders the top three prices, and so forth. We call this a "switch-over" policy. 
It is important to note that the switch-over policy essentially follows the time epochs $t_{\ell}(d)$ $(\ell=1, \ldots, m)$ in Proposition 1 but ignores their dependence on the inventory level $d$. Consequently, it is suboptimal even in the context of Proposition 11.

In this section, we focus on the case of a constant (unit) batch size, treating the general case of random batch sizes in the next section.

\subsection{Optimizing the Switch-Over Times}

We let the switch-over times be the decision variables of an optimization problem, with the objective to maximize the expected profit over the horizon - the same objective as in the dynamic programming formulation. (Note, however, since these time epochs are chosen independently of the level of the available inventory, the switch-over policy is in general sub-optimal.) To determine the best switch-over times,

$$
0=t_{0} \leq t_{1} \leq t_{2} \leq \cdots \leq t_{m-1} \leq t_{m}=T
$$

(only $t_{1}, \ldots, t_{m-1}$ are decision variables), we consider a continuous-time version of the original problem, with the order streams following independent Poisson processes with rates $\lambda_{i}, i=$ $1, \ldots, m$, and associated with the price $p_{i}$, such that

$$
p_{1} \geq p_{2} \geq \cdots \geq p_{m}
$$

Let $N_{1 k}$ denote the total number of order arrivals of types $\{1,2, \cdots, k\}$ over the time interval $\left(t_{k-1}, t_{k}\right] ; N_{1 k}$ follows a Poisson distribution with mean $\left(\lambda_{1}+\cdots+\lambda_{k}\right)\left(t_{k}-t_{k-1}\right)$. Let

$$
p_{1 k}:=\frac{\lambda_{1} p_{1}+\cdots+\lambda_{k} p_{k}}{\lambda_{1}+\cdots+\lambda_{k}}
$$

denote the average unit price of the orders accepted (by the switch-over policy) over the time interval $\left(t_{k-1}, t_{k}\right]$. Then, we can write the objective function associated with the switch-over policy as follows:

$$
\begin{aligned}
\max & p_{11} \mathrm{E}\left[W \wedge N_{1}\right]+p_{12} \mathrm{E}\left[\left(W-N_{11}\right)^{+} \wedge N_{12}\right] \\
& +\cdots+p_{1 m} \mathrm{E}\left[\left(W-\sum_{k=1}^{m-1} N_{1 k}\right)^{+} \wedge N_{1 m}\right] \\
= & p_{11}\left[W-\mathrm{E}\left(W-N_{11}\right)^{+}\right]+p_{12}\left[\mathrm{E}\left(W-N_{11}\right)^{+}-\mathrm{E}\left(W-N_{1}-N_{12}\right)^{+}\right] \\
& +\cdots+p_{1 m}\left[\mathrm{E}\left(W-\sum_{k=1}^{m-1} N_{1 k}\right)^{+}-\mathrm{E}\left(W-\sum_{k=1}^{m} N_{1 k}\right)^{+}\right] \\
= & p_{11} W-\left(p_{11}-p_{12}\right) \mathrm{E}\left(W-N_{11}\right)^{+}-\cdots-\left(p_{1, m-1}-p_{1 m}\right) \mathrm{E}\left(W-\sum_{k=1}^{m-1} N_{1 k}\right)^{+} \\
& -p_{1 m} \mathrm{E}\left(W-\sum_{k=1}^{m} N_{1 k}\right)^{+}
\end{aligned}
$$


Denote

$$
\begin{aligned}
\mu_{k} & :=\lambda_{1} t_{k}+\lambda_{2}\left(t_{k}-t_{1}\right)+\cdots+\lambda_{k}\left(t_{k}-t_{k-1}\right) \\
& =\lambda_{1} t_{1}+\left(\lambda_{1}+\lambda_{2}\right)\left(t_{2}-t_{1}\right)+\cdots+\left(\lambda_{1}+\cdots+\lambda_{k}\right)\left(t_{k}-t_{k-1}\right),
\end{aligned}
$$

which is the mean (as well as the variance) of $N_{11}+N_{12}+\cdots+N_{1 k}$. Let $N(\mu)$ denote a Poisson random variable with mean $\mu$. We can then turn the optimization problem in (9) into the following equivalent form:

$$
\begin{array}{ll}
\min _{0 \leq t_{1} \leq \cdots \leq t_{m-1} \leq T} & \left(p_{11}-p_{12}\right) \mathrm{E}\left[W-N\left(\mu_{1}\right)\right]^{+}+\left(p_{12}-p_{13}\right) \mathrm{E}\left[W-N\left(\mu_{2}\right)\right]^{+}+\cdots \\
& +\left(p_{1, m-1}-p_{1, m}\right) \mathrm{E}\left[W-N\left(\mu_{m-1}\right)\right]^{+}+p_{1 m} \mathrm{E}\left[W-N\left(\mu_{m}\right)\right]^{+} .
\end{array}
$$

Denote the distribution function of $N(\mu)$ as:

$$
F_{n}(\mu):=\mathrm{P}[N(\mu) \leq n]=\sum_{k=0}^{n} \frac{\mu^{k}}{k !} e^{-\mu}
$$

and define a function:

$$
H(\mu):=\mathrm{E}[W-N(\mu)]^{+}=\sum_{k=0}^{W}(W-k) \frac{\mu^{k}}{k !} e^{-\mu}=W F_{W}(\mu)-\mu F_{W-1}(\mu) .
$$

Clearly, $H(\mu)$ is decreasing and convex in $\mu$, since $(x)^{+}$is increasing and convex, and $N(\mu)$ is stochastically increasing and linear in $\mu$ (refer to [18]).

From (13), we can derive explicitly $H^{\prime}\left(\mu_{\ell}\right)$, which will become useful below. First, taking the derivative of $F_{n}(\mu)$ in (12), we have,

$$
-f_{n}(\mu):=F_{n}^{\prime}(\mu)=F_{n-1}(\mu)-F_{n}(\mu)=-\frac{\mu^{n}}{n !} e^{-\mu} .
$$

Hence

$$
H^{\prime}(\mu)=-W f_{W}(\mu)-F_{W-1}(\mu)+\mu f_{W-1}(\mu)=-F_{W-1}(\mu)
$$

taking into account that $f_{W}(\mu)=\frac{\mu}{W} f_{W-1}(\mu)$. As a by-product, the above also leads to

$$
H^{\prime \prime}\left(\mu_{\ell}\right)=f_{W-1}\left(\mu_{\ell}\right) \geq 0,
$$

confirming the convexity of $H(\mu)$.

Denote:

$$
\pi_{k}:=p_{1 k}-p_{1, k+1}, \quad y_{k}:=t_{k}-t_{k-1}, \quad k=1, \ldots, m
$$


with $p_{1, m+1}:=0$, and recall $t_{0}:=0$ and $t_{m}:=T$. Then, the optimization problem in (11) can be expressed as follows:

$$
\min _{\left(y_{\ell}\right)} \sum_{\ell=1}^{m} \pi_{\ell} H\left(\mu_{\ell}\right) \quad \text { s.t. } \quad \sum_{\ell=1}^{m} y_{\ell}=T ; \quad y_{\ell} \geq 0, \quad \ell=1, \ldots, m \text {; }
$$

where the new decision variables are $\left(y_{\ell}\right)$; and, following (10) and (16),

$$
\mu_{\ell}=\Lambda_{1} y_{1}+\Lambda_{2} y_{2}+\cdots+\Lambda_{\ell} y_{\ell}
$$

with

$$
\Lambda_{\ell}:=\lambda_{1}+\cdots+\lambda_{\ell}
$$

\subsection{Solution Approach}

A shortfall of the above formulation is that the objective function in (17) is not separable in $\left(y_{\ell}\right)$ (although the constraint is). If we make $\left(\mu_{\ell}\right)$ the decision variables instead, then both the objective and the constraint will become separable. From (18), we can solve for $\left(y_{\ell}\right)$ :

$$
y_{\ell}=\frac{\mu_{\ell}-\mu_{\ell-1}}{\Lambda_{\ell}}, \quad \ell=1, \ldots, m
$$

with $\mu_{0}:=0$. We can then turn the optimization problem in (17) into one with $\left(\mu_{\ell}\right)$ as decision variables as follows:

$$
\begin{array}{ll}
\min _{\left(\mu_{\ell}\right)} & \sum_{\ell=1}^{m} \pi_{\ell} H\left(\mu_{\ell}\right) \\
\text { s.t. } & \sum_{\ell=1}^{m}\left(\frac{1}{\Lambda_{\ell}}-\frac{1}{\Lambda_{\ell+1}}\right) \mu_{\ell} \leq T, \\
& 0 \leq \mu_{1} \leq \cdots \leq \mu_{m} ;
\end{array}
$$

where $\Lambda_{m+1}^{-1}:=0$. Also note that in (22) we have changed the original equality constraint $(=T)$ to an inequality constraint $(\leq T)$, taking into account that $H(\cdot)$ is a decreasing function, and $\pi_{\ell} \geq 0$ as evident from (7, 816). Furthermore, as pointed out earlier, $H(\cdot)$ is convex; hence, we now have a separable convex programming problem. In addition, it has another appealing property as revealed in the lemma below.

Lemma 2 The ratio of the coefficients in (22) to those in (21), $\left(\frac{1}{\Lambda_{\ell}}-\frac{1}{\Lambda_{\ell+1}}\right) / \pi_{\ell}$, is decreasing in $\ell$.

Proof. We need to show:

$$
\frac{1}{\pi_{\ell-1}}\left(\frac{1}{\Lambda_{\ell-1}}-\frac{1}{\Lambda_{\ell}}\right) \geq \frac{1}{\pi_{\ell}}\left(\frac{1}{\Lambda_{\ell}}-\frac{1}{\Lambda_{\ell+1}}\right), \quad \ell=2, \ldots, m .
$$


The above inequalities simplify to:

$$
\frac{\pi_{\ell}}{\pi_{\ell-1}} \frac{\lambda_{\ell}}{\lambda_{\ell+1}} \geq \frac{\Lambda_{\ell-1}}{\Lambda_{\ell+1}}, \quad \ell=2, \ldots, m-1 ; \quad \text { and } \quad \frac{\pi_{m}}{\pi_{m-1}} \frac{\lambda_{m}}{\Lambda_{m-1}} \geq 1
$$

To verify these inequalities, write

$$
\rho_{\ell}:=\sum_{k=1}^{\ell} \lambda_{k} p_{k} \quad \text { and } \quad \pi_{\ell}=\frac{\rho_{\ell}}{\Lambda_{\ell}} .
$$

First consider $\ell \neq m$. Straightforward algebra yields:

$$
\begin{aligned}
\frac{\pi_{\ell} \Lambda_{\ell+1}}{\pi_{\ell-1} \Lambda_{\ell-1}} & =\frac{\rho_{\ell} \Lambda_{\ell+1}-\rho_{\ell+1} \Lambda_{\ell}}{\rho_{\ell-1} \Lambda_{\ell}-\rho_{\ell} \Lambda_{\ell-1}} \\
& =\frac{\rho_{\ell} \lambda_{\ell+1}-p_{\ell+1} \lambda_{\ell+1} \Lambda_{\ell}}{\rho_{\ell-1} \lambda_{\ell}-p_{\ell} \lambda_{\ell} \Lambda_{\ell-1}} .
\end{aligned}
$$

We want the above to dominate $\lambda_{\ell+1} / \lambda_{\ell}$. This is equivalent to

$$
\rho_{\ell}-p_{\ell+1} \Lambda_{\ell} \geq \rho_{\ell-1}-p_{\ell} \Lambda_{\ell-1}
$$

or,

$$
p_{\ell} \lambda_{\ell} \geq p_{\ell+1} \Lambda_{\ell}-p_{\ell} \Lambda_{\ell-1}=p_{\ell} \lambda_{\ell}-\left(p_{\ell}-p_{\ell+1}\right) \Lambda_{\ell}
$$

which obviously holds, since $p_{\ell} \geq p_{\ell+1}$. Next, we show the last inequality in (24). Since

$$
\pi_{m}=\frac{\rho_{m}}{\Lambda_{m}}, \quad \pi_{m-1}=\frac{\rho_{m-1}}{\Lambda_{m-1}}-\frac{\rho_{m}}{\Lambda_{m}}
$$

we have

$$
\frac{\pi_{m}}{\pi_{m-1}}=\frac{\rho_{m} \Lambda_{m-1}}{\rho_{m-1} \Lambda_{m}-\rho_{m} \Lambda_{m-1}} .
$$

We want the above to dominate $\frac{\Lambda_{m-1}}{\lambda_{m}}$; and this simplifies to

$$
\rho_{m} \lambda_{m} \geq \rho_{m-1} \Lambda_{m}-\rho_{m} \Lambda_{m-1}
$$

or $\rho_{m} \Lambda_{m} \geq \rho_{m-1} \Lambda_{m}$, i.e., $\rho_{m} \geq \rho_{m-1}$, which certainly holds.

The above lemma suggests an algorithm as follows. Ignoring the constraint in (23), and with $\eta \geq 0$ denoting the Lagrangian multiplier associated with the constraint in (22), we have the following optimality equations:

$$
F_{W-1}\left(\mu_{\ell}\right)=\frac{\eta}{\pi_{\ell}}\left(\frac{1}{\Lambda_{\ell}}-\frac{1}{\Lambda_{\ell+1}}\right), \quad \ell=1, \ldots, m .
$$

(Recall from (15), we have $F_{W-1}(\mu)=-H^{\prime}(\mu)$.) From (14), we know $F_{W-1}(\mu)$ is decreasing in $\mu$; and from Lemma 2, we know the right hand sides of the equations above are also decreasing in $\ell$. Hence, the solution to these optimality equations, $\mu_{\ell}$, must be increasing in $\ell$. 
To solve these equations, we can start with an $\eta$ value sufficiently close to zero, so that each of the $m$ equations in (25) has a solution. (Note that $F_{W-1}(\mu) \downarrow 0$ when $\mu \rightarrow \infty$.) This may very well violate the constraint in (22), as the resource is priced too low. Hence, we will gradually increase $\eta$, to bring down the left hand side of the constraint in (22), until it is satisfied as an equality. (Note that since $F_{W-1}(\mu)$ is decreasing in $\mu$, increasing $\eta$ will decrease the value of $\mu_{\ell}$ 's that solve the equation in (25).) As we increase $\eta$, it can happen that for some $k$, even with $\mu_{k}=0$, we still have

$$
R_{k}:=\frac{F_{W-1}(0)}{\frac{1}{\pi_{k}}\left(\frac{1}{\Lambda_{k}}-\frac{1}{\Lambda_{k+1}}\right)}<\eta .
$$

Then, we set $\mu_{k}=0$, as any $\mu_{k}>0$ will only lead to $F_{W-1}\left(\mu_{k}\right)<F_{W-1}(0)$. In this case we also set $\mu_{j}=0$ for all $j<k$ as well, since $R_{j} \leq R_{k}$ following Lemma 2, Furthermore, $\mu_{1}=\cdots=\mu_{k}=0$ will remain at zero as $\eta$ further increases. (As $\eta$ increases, more $\mu_{\ell}$ 's may become zero, for $\ell>k$.)

Proposition 3 (i) The optimal switch-over times can be obtained from solving the set of singlevariable equations in (25) along with an increasing sequence of $\eta$ (the Lagrangian multiplier) until the constraint in (22) becomes binding.

(ii) At optimality, for any price class $k$ we have $\mu_{k}=0$ if and only if $R_{k}<\eta$ in (26); and if $\mu_{k}=0$ for some $k$, we must have $\mu_{j}=0$ for all $j \leq k$.

Proof. What is stated in (i) and (ii) is a summary of the algorithm and the solution it generates. Hence, what needs to be argued is the optimality of the solution. To do so, in addition to the Lagrangian multiplier $\eta \geq 0$, let $\nu_{\ell} \geq 0$ be the Lagrangian multiplier associated with the constraint $\mu_{\ell} \geq \mu_{\ell-1}$, for $\ell=1, \ldots, m$ (with $\mu_{0}:=0$ ). In addition, denote $\nu_{m+1}:=0$. Then, optimality is reached if and only if $\left(\mu_{1}, \ldots, \mu_{m}\right)$ and all the multipliers satisfy the following equations:

$$
\begin{aligned}
& \pi_{\ell} F_{W-1}\left(\mu_{\ell}\right)=\eta\left(\frac{1}{\Lambda_{\ell}}-\frac{1}{\Lambda_{\ell+1}}\right)-\nu_{\ell}+\nu_{\ell+1}, \quad \ell=1, \ldots, m \\
& \eta\left[\sum_{\ell=1}^{m}\left(\frac{1}{\Lambda_{\ell}}-\frac{1}{\Lambda_{\ell+1}}\right) \mu_{\ell}-T\right]=0 \\
& \nu_{\ell}\left(\mu_{\ell}-\mu_{\ell-1}\right)=0, \quad \ell=1, \ldots, m
\end{aligned}
$$

and the constraints in (22) and (23) are satisfied.

As described above, when the algorithm ends, it generates a solution that takes the following form:

$$
0:=\mu_{0}=\mu_{1}=\cdots=\mu_{k}<\mu_{k+1} \leq \mu_{k+2} \leq \cdots \leq \mu_{m}
$$


for some $k \in\{1, \ldots, m\}$, along with an $\eta \geq 0$, while (22) is satisfied as an equality - and hence (28) holds. In addition, (23) follows from (30). Hence, what remains is to verify (27) and (29). These can be satisfied by letting

$$
\begin{aligned}
\nu_{\ell} & =0, \quad \ell=k+1, \ldots, m \\
\nu_{k} & =\eta\left(\frac{1}{\Lambda_{k}}-\frac{1}{\Lambda_{k+1}}\right)-\pi_{k} F_{W-1}(0) \\
\nu_{j} & =\nu_{j+1}+\eta\left(\frac{1}{\Lambda_{j}}-\frac{1}{\Lambda_{j+1}}\right)-\pi_{j} F_{W-1}(0), \quad j=k-1, \ldots, 1 .
\end{aligned}
$$

First, the $\nu$ 's above are all non-negative: $\nu_{k}>0$ follows from (26); and for $j<k, \nu_{j}>0$ follows recursively from (33), as well as from (26), taking into account $R_{j} \leq R_{k}<\eta$ for $j<k$. Second, the above $\nu$ 's, along with the $\mu$ 's in (30), satisfy (29). Finally, note that the zero $\nu_{\ell}$ 's $(\ell=k+1, \ldots, m)$ in (31) reduce (27) to the equations (with the same $\ell$ indices) in (25) used by the algorithm; and substituting (32) and (33) into (27) recovers $\mu_{j}=0$ obtained by the algorithm for $j \leq k$.

\section{The Switch-Over Policy: Random Batch Size}

We now extend the switch-over policy of the last section to allow random batch sizes. In this case, while the structural properties in Proposition 1 do not apply, the asymptotic optimality results in the next section does provide some justification to the switch-over policy.

\subsection{Homogeneous Batches}

To facilitate the derivation, we start with assuming that all orders independently follow an identical batch-size distribution that is independent of the prices, deferring the case of pricedependent batch sizes to the next subsection. Specifically, the batch size distribution is denoted by $q_{j}=\mathrm{P}\{Q=j\}$, for $j=1, \ldots, W$.

With the initial inventory of $W$ units, we apply the switch-over policy in $\left[0, t_{1}\right]$. The cumulative revenue collected by time $t_{1}$ is:

$$
p_{1} \mathrm{E}\left[W-\mathbf{z}^{T} M^{N_{1}} \mathbf{w}\right]
$$

where,

$$
\mathbf{z}^{T}:=(0,0, \ldots, 0,1), \quad \mathbf{w}^{T}:=(0,1,2, \ldots, W) ;
$$

$N_{1}:=N_{1}\left(t_{1}\right)$ follows the Poisson distribution with mean $\lambda_{1} t_{1}$, and $M$ denotes the following 
probability transition matrix:

$$
M=\left(\begin{array}{ccccc}
1 & 0 & 0 & \ldots & 0 \\
q_{1} & 1-q_{1} & 0 & \ldots & 0 \\
q_{2} & q_{1} & 1-q_{1}-q_{2} & \ldots & 0 \\
& & & \ldots & \\
q_{W} & q_{W-1} & q_{W-2} & \ldots & 1-q_{1}-\ldots-q_{W}
\end{array}\right)
$$

That is, the number of remaining units in the system, embedded at the arrival epochs of orders of price $p_{1}$, is a Markov chain with the probability transition matrix $M$. Note that the dimension of $M$ is $W+1$, corresponding to the dimension of the state space of the Markov chain, $\{0,1, \ldots, W\}$.

Recall that the generating function of a Poisson variable $N$ with mean $\mu$ is:

$$
\mathrm{E}\left[z^{N}\right]=e^{-\mu} e^{\mu z}
$$

Hence, we have

$$
p_{1} \mathrm{E}\left[W-\mathbf{z}^{T} M^{N_{1}} \mathbf{w}\right]=p_{1}\left[W-e^{-\mu_{1}} \mathbf{z}^{T} e^{\mu_{1} M} \mathbf{w}\right]
$$

Here, $\mu_{1}$ follows the notation defined in (18); and the matrix exponent is defined as

$$
e^{A}=\sum_{j=0}^{\infty} \frac{A^{k}}{k !},
$$

for any matrix $A$.

Similarly, for the next interval $\left(t_{1}, t_{2}\right]$, when the acceptance includes both price classes $p_{1}$ and $p_{2}$, the expected revenue is (using the notation of the last section):

$$
\begin{aligned}
& p_{12} \mathrm{E}\left[\mathbf{z}^{T} M^{N_{1}} \mathbf{w}-\mathbf{z}^{T} M^{N_{1}+N_{12}} \mathbf{w}\right] \\
= & p_{12}\left[\mathbf{z}^{T}\left(e^{-\mu_{1}} e^{\mu_{1} M}-e^{-\mu_{2}} e^{\mu_{2} M}\right) \mathbf{w}\right] .
\end{aligned}
$$

Comparing the above with the objective function in (9), we know the only difference is that the terms there,

$$
\mathrm{E}\left(W-N_{1}\right)^{+} \text {and } \mathrm{E}\left(W-N_{1}-N_{12}\right)^{+}
$$

are now replaced by

$$
\mathbf{z}^{T} e^{-\mu_{1}} e^{\mu_{1} M} \mathbf{w} \quad \text { and } \quad \mathbf{z}^{T} e^{-\mu_{2}} e^{\mu_{2} M} \mathbf{w}
$$

With this in mind, we further denote

$$
G(\mu):=\mathbf{z}^{T} e^{-\mu} e^{\mu M} \mathbf{w}
$$


which is analogous to $H(\mu)$ of the last section. The derivatives of $G(\mu)$ can be derived as follows:

$$
G^{\prime}(\mu)=-\mathbf{z}^{T} e^{-\mu}(I-M) e^{\mu M} \mathbf{w}, \quad G^{\prime \prime}(\mu)=\mathbf{z}^{T} e^{-\mu}(I-M)^{2} e^{\mu M} \mathbf{w} .
$$

Note that the spectral radius of the matrix $M$ is unity. Hence, $G^{\prime}(\mu) \leq 0$; i.e., $G(\mu)$ is a decreasing function. Furthermore, $G(\mu)$ is a convex function, since $G^{\prime \prime}(\mu) \geq 0$.

We can formulate the optimization problem as follows, with $\mu_{\ell}, \ell=1, \ldots, m$, as decision variables:

$$
\min _{\left(\mu_{\ell}\right)} \sum_{\ell=1}^{m} \pi_{\ell} G\left(\mu_{\ell}\right) ; \quad \text { s.t. } \sum_{\ell=1}^{m}\left(\frac{1}{\Lambda_{\ell}}-\frac{1}{\Lambda_{\ell+1}}\right) \mu_{\ell} \leq T, \quad 0 \leq \mu_{1} \leq \cdots \leq \mu_{m} .
$$

Here, same as in (20),

$$
y_{\ell}:=t_{\ell}-t_{\ell-1}=\frac{\mu_{\ell}-\mu_{\ell-1}}{\Lambda_{\ell}}, \quad \ell=1, \ldots, m
$$

with $\mu_{0}:=0$.

To solve the optimization problem above, we can follow the same approach as in the last section; specifically, we can solve the following optimality equations via gradually increasing the Lagrangian multiplier $\eta$ until the resource constraint becomes binding:

$$
\mathbf{z}^{T} e^{-\mu_{\ell}}(I-M) e^{\mu_{\ell} M} \mathbf{w}=\frac{\eta}{\pi_{\ell}}\left(\frac{1}{\Lambda_{\ell}}-\frac{1}{\Lambda_{\ell+1}}\right), \quad \ell=1, \ldots, m .
$$

Note that both Lemma 2 and Proposition 3 still apply here.

\subsection{Price-Dependent Batches}

Now, suppose each price $p_{i}, i=1, \ldots, m$, is associated with a batch size $Q_{i}$, with the distribution $\mathrm{P}\left(Q_{i}=j\right)=\theta_{i j}$, for $j=1, \ldots, W$. The batch sizes are i.i.d. among orders of the same price, and independent among orders of different prices. Here, instead of a single probability transition matrix $M$, we have $m$ such matrices, one for each price $p_{i}$ :

$$
M_{i}=\left(\begin{array}{ccccc}
1 & 0 & 0 & \ldots & 0 \\
\theta_{i 1} & 1-\theta_{i 1} & 0 & \ldots & 0 \\
\theta_{i 2} & \theta_{i 1} & 1-\theta_{i 1}-\theta_{i 2} & \ldots & 0 \\
& & & \ldots & \\
\theta_{i W} & \theta_{i, W-1} & \theta_{i, W-2} & \ldots & 1-\theta_{i 1}-\ldots-\theta_{i W}
\end{array}\right) .
$$

Consider the second time interval $\left(t_{1}, t_{2}\right]$. Since orders of both prices $p_{1}$ and $p_{2}$ are accepted, the two Poisson streams will be combined, resulting in the following transition matrix

$$
\Gamma_{2}:=\frac{1}{\Lambda_{2}}\left(\lambda_{1} M_{1}+\lambda_{2} M_{2}\right)
$$


Analogously, denote $\Gamma_{1}:=M_{1}$. The expected revenue over this time interval is

$$
\begin{aligned}
& p_{12} \mathrm{E}\left[\mathbf{z}^{T} M_{1}^{N_{1}} \mathbf{w}-\mathbf{z}^{T} M_{1}^{N_{1}} \Gamma_{2}^{N_{12}} \mathbf{w}\right] \\
= & p_{12}\left[\mathbf{z}^{T}\left(e^{-\mu_{1}} e^{\mu_{1} \Gamma_{1}}-e^{-\mu_{2}} e^{\mu_{1} \Gamma_{1}} e^{\left(\mu_{2}-\mu_{1}\right) \Gamma_{2}}\right) \mathbf{w}\right] .
\end{aligned}
$$

In general, denote

$$
\Gamma_{\ell}:=\sum_{j=1}^{\ell} \frac{\lambda_{j}}{\Lambda_{\ell}} M_{j}
$$

Replace $G\left(\mu_{\ell}\right)$ in the earlier special case of independent batch sizes by the following:

$$
g_{\ell}\left(\mathbf{y}_{\ell}\right):=e^{-\mu_{\ell}} \exp \left(\sum_{k=1}^{\ell} \Lambda_{k} y_{k} \Gamma_{k}\right) \quad \text { and } \quad G_{\ell}\left(\mathbf{y}_{\ell}\right):=\mathbf{z}^{T} g_{\ell}\left(\mathbf{y}_{\ell}\right) \mathbf{w} .
$$

(Notice that from (18), we have $\mu_{\ell}-\mu_{\ell-1}=\Lambda_{\ell} y_{\ell}$.)

The optimization problem can now be expressed as follows, with $y_{\ell}, \ell=1, \ldots, m$, as decision variables:

$$
\min _{y} \sum_{\ell=1}^{m} \pi_{\ell} G_{\ell}\left(\mathbf{y}_{\ell}\right) ; \quad \text { s.t. } \sum_{\ell=1}^{m} y_{\ell} \leq T ; \quad y_{\ell} \geq 0, \quad \ell=1, \ldots, m .
$$

From (40), we know that $g_{\ell}\left(\mathbf{y}_{\ell}\right)$ is convex in $\mathbf{y}$; hence, the problem in (41) is a convex program (albeit no longer separable) and as such can be solved by standard algorithms.

\subsection{A Numerical Example}

Example 1 Consider four price classes:

$$
p_{1}=1, \quad p_{2}=0.8, \quad p_{3}=0.65, \quad p_{4}=0.45,
$$

with Poisson arrivals at the following rates:

$$
\lambda_{1}=0.2, \quad \lambda_{2}=0.3, \quad \lambda_{3}=0.1, \quad \lambda_{4}=0.4 .
$$

The batch size $Q$ is homogeneous among the four classes, following a discretized exponential distribution with mean 12. (Specifically, $\mathrm{P}(Q=n)=e^{\lambda(n+1)}-e^{\lambda n}$, for $n=0,1,2 \ldots$, and $1 / \lambda=12$.) Let $T=20$, and let $W$ vary from 1 to 100 .

We compare the performance of the switch-over policy against the optimal policy (from DP) and a first-come-first-served (FCFS) policy (i.e. supply any order on arrival as long as there are units available). The objective values corresponding to the three policies are plotted

in Figure 1. The relative errors of the switch-over policy and the FCFS policy with respect to the optimal policies are plotted in Figure 2. The performance of the switch-over policy is remarkably close to the optimal policy, while the performance of the FCFS policy, as expected, deteriorates quickly as $W$ increases. 


\section{$5 \quad$ Asymptotic Optimality of the Switch-Over Policy}

In this section, we establish the fact that the switch-over policy is asymptotically optimal, in the sense that as $W$ increases to infinity, the relative error between the switch-over policy and the optimal policy (via dynamic programming) will go to zero. In fact, we show the absolute error 
is no more than $O(\sqrt{W})$; hence, the rate of convergence is $O(\sqrt{W})$. This type of asymptotic optimality is in the same spirit as other recent studies, e.g., [14. Similar results have been established for various revenue management problems, see, e.g. [3, 9, 19]. The result here applies to both models in the last two sections; for ease of exposition, we shall focus on the model in 93 .

Intuitively, when the available inventory $W \rightarrow \infty$, we should expect the switch-over policy to be optimal in the following two cases: (a) if the decision horizon $T$ remains a constant or is of lower-order than $W$, then supplying all orders (i.e., first-come-first-served) would be optimal; and (b) if $T$ is of higher order than $W$, then supplying the highest bid only (orders priced at $\left.p_{1}\right)$ should be optimal. In both cases the policies can be represented as special cases of the switch-over policy. Below, we will formally confirm the intuition behind these two special cases, and also prove the result in the non-trivial case in which $T \rightarrow \infty$ in the same order as $W$.

We start from an upper-bound on the performance of the optimal policy.

Lemma 4 The objective value under the optimal policy (as derived from the dynamic programming), denoted $V^{*}$, satisfies the following inequality:

$$
V^{*} \leq\left(\lambda_{1} p_{1}+\cdots+\lambda_{k} p_{k}\right) T+\lambda_{k+1} p_{k+1} t
$$

where $k=1, \ldots, m-1$ and $t \in[0, T)$ are such that $\Lambda_{k} T \leq W, \Lambda_{k+1} T>W$, and

$$
\Lambda_{k} T+\lambda_{k+1} t=W
$$

If $\Lambda_{1} T>W$, then $V^{*} \leq p_{1} W$. If $\Lambda_{m} T \leq W$, then $V^{*} \leq p_{1 m} \Lambda_{m} T$.

Proof. Note that $V^{*}$ is maximal among all non-anticipative policies; in particular, at each time $t$ all future order arrivals and their prices are only known in distribution. Now, suppose at time $t=0$ we know all arrivals over $(0, T]$ and their prices deterministically. Specifically, suppose over (0.T], there are $N_{1}$ orders of price $p_{1}, N_{2}$ orders of price $p_{2}, \ldots$, and $N_{m}$ orders of price $p_{m}$. Then, we will obviously use the $W$ available units to first supply the $N_{1}$ orders of price $p_{1}$; and then, if there is anything left, supply the $N_{2}$ orders of price $p_{2}$; and so forth. Clearly, no non-anticipative policy can do better than this one, which yields an objective value of

$$
\begin{aligned}
& p_{1}\left[W \wedge N_{1}\right]+p_{2}\left[\left(W-N_{1}\right)^{+} \wedge N_{2}\right]+\cdots+p_{m}\left[\left(W-\sum_{k=1}^{m-1} N_{k}\right)^{+} \wedge N_{m}\right] \\
= & p_{1} W-\left(p_{1}-p_{2}\right)\left(W-N_{1}\right)^{+}-\cdots-\left(p_{m-1}-p_{m}\right)\left(W-\sum_{k=1}^{m-1} N_{k}\right)^{+}-p_{m}\left(W-\sum_{k=1}^{m} N_{k}\right)^{+} .
\end{aligned}
$$


Taking expectation on the right hand side above and passing the expectation into $(\cdot)^{+}$further increases its value (since the function $(x)^{+}$is convex). Hence, we have

$$
\begin{aligned}
V^{*} \leq & p_{1} W-\left(p_{1}-p_{2}\right)\left(W-\lambda_{1} T\right)^{+}-\cdots-\left(p_{m-1}-p_{m}\right)\left(W-\sum_{k=1}^{m-1} \lambda_{k} T\right)^{+} \\
& -p_{m}\left(W-\sum_{k=1}^{m-1} \lambda_{k} T\right)^{+} \\
= & p_{1} W-\left(p_{1}-p_{2}\right)\left(W-\lambda_{1} T\right)-\cdots-\left(p_{k}-p_{k+1}\right)\left(W-\lambda_{1} T-\cdots-\lambda_{k} T\right) \\
= & p_{k+1} W+\left(p_{1}-p_{k+1}\right) \lambda_{1} T+\left(p_{2}-p_{k+1}\right) \lambda_{2} T+\cdots+\left(p_{k}-p_{k+1}\right) \lambda_{k} T \\
= & \left(\lambda_{1} p_{1}+\cdots+\lambda_{k} p_{k}\right) T+\lambda_{k+1} p_{k+1} t
\end{aligned}
$$

where the last two equalities follow from the relation in (43).

Following the same reasoning also leads to the bounds in the two cases when $\Lambda_{1} T>W$ or $\Lambda_{m} T \leq W$.

Next, we derive a lower bound on the switch-over policy.

Lemma 5 Let $V^{\text {sw }}$ denote the objective value under the best switch-over policy. Then, letting $k$ and $t$ be defined as in Lemma 4, we have

$$
\begin{aligned}
V^{\mathrm{sw}} \geq & p_{1 k} W-\left(p_{1 k}-p_{1, k+1}\right) \mathrm{E}\left[W-N\left(\Lambda_{k}(T-t)\right)\right]^{+} \\
& -p_{1, k+1} \mathrm{E}\left[W-N\left(\Lambda_{k} T+\lambda_{k+1} t\right)\right]^{+}
\end{aligned}
$$

and

$$
\begin{aligned}
V^{\mathrm{sw}} \geq p_{1} \mathrm{E}[N(W) \wedge W] \quad \text { if } & \Lambda_{1} T>W, \\
V^{\mathrm{sw}} \geq p_{1 m} \mathrm{E}\left[N\left(\Lambda_{m} T\right) \wedge W\right] \quad \text { if } & \Lambda_{m} T \leq W .
\end{aligned}
$$

Proof. Clearly, a feasible switch-over policy, realizable by setting $t_{1}=\cdots=t_{k}=0$ and $t_{k+1}=T-t$, is to accept the top $k$ price classes throughout the horizon $[0, T]$ and the class $k+1$ over $[T-t, T]$, the last $t$ time units. Hence,

$$
\begin{aligned}
V^{\mathrm{sw}} \geq & p_{1 k} \mathrm{E}\left[N\left(\Lambda_{k}(T-t)\right) \wedge W\right]+p_{1, k+1} \mathrm{E}\left\{N\left(\Lambda_{k+1} t\right) \wedge\left[W-N\left(\Lambda_{k}(T-t)\right)\right]^{+}\right\} \\
= & p_{1 k} W-p_{1 k} \mathrm{E}\left[W-N\left(\Lambda_{k}(T-t)\right)\right]^{+} \\
& +p_{1, k+1} \mathrm{E}\left[W-N\left(\Lambda_{k}(T-t)\right)\right]^{+}-p_{1, k+1} \mathrm{E}\left[W-N\left(\Lambda_{k}(T-t)\right)-N\left(\Lambda_{k+1} t\right)\right]^{+} \\
= & p_{1 k} W-\left(p_{1 k}-p_{1, k+1}\right) \mathrm{E}\left[W-N\left(\Lambda_{k}(T-t)\right)\right]^{+}-p_{1, k+1} \mathrm{E}\left[W-N\left(\Lambda_{k} T+\lambda_{k+1} t\right)\right]^{+}
\end{aligned}
$$


(Recall, $p_{1 k}$ is the average price among classes 1 through $k$, defined in (8), and $p_{1, k+1}$ is similarly defined.) If $\Lambda_{1} T>W$, then serving class 1 only throughout $[0, T]$ results in

$$
V^{\mathrm{sw}} \geq p_{1} \mathrm{E}\left[N\left(\Lambda_{1} T\right) \wedge W\right] \geq p_{1} \mathrm{E}[N(W) \wedge W]
$$

If $\Lambda_{m} T \leq W$, then serving all $m$ classes throughout $[0, T]$ leads to $V^{\mathrm{sw}} \geq p_{1 m} \mathrm{E}\left[N\left(\Lambda_{m} T\right) \wedge W\right]$.

We shall also need the following result: For the Poisson variate with mean $a$, denoted $N(a)$,

$$
\mathrm{E}[a-N(a)]^{+} \sim \sqrt{\frac{a}{2 \pi}}, \quad a \rightarrow \infty ;
$$

which can be directly verified via the Poisson distribution (along with the Stirling formula), or via the normal approximation of the Poisson distribution.

Proposition 6 The switch-over policy is asymptotically optimal, in the sense that $V^{\mathrm{sw}} / V^{*} \rightarrow$ 1 when $W \rightarrow \infty$.

Proof. To start with, suppose while $W \rightarrow \infty, T$ remains a constant. This corresponds to the case of $\Lambda_{m} T<W$ in Lemmas 4 and 5, and we have

$$
\begin{aligned}
p_{1 m} \Lambda_{m} T \geq V^{*} \geq V^{\mathrm{SW}} & \geq p_{1 m} \mathrm{E}\left[N\left(\Lambda_{m} T\right) \wedge W\right] \\
& \rightarrow p_{1 m} \mathrm{E}\left[N\left(\Lambda_{m} T\right)=p_{1 m} \Lambda_{m} T,\right.
\end{aligned}
$$

where the limit follows from monotone convergence as $W \rightarrow \infty$. Hence, in this case we have $V^{*}=V^{\mathrm{SW}}=p_{1 m} \Lambda_{m} T$.

Now, suppose $T \rightarrow \infty$; and without loss of generality, suppose as a function of $W, T(W)$ satisfies the following:

$$
0 \leq c:=\liminf \frac{T(W)}{W} \leq \infty .
$$

That is, we allow $T$ to be of lower or higher order than $W$, as well as of equal order to $W$. First, suppose $\Lambda_{1}^{-1} \leq c \leq \Lambda_{m}^{-1}$. Then, we can assume the relation between $W$ and $T$ in (43) to hold, for some $k$. Note that the upper bound in (42) can be written as:

$$
\begin{aligned}
& \left(\lambda_{1} p_{1}+\cdots+\lambda_{k} p_{k}\right) T+\lambda_{k+1} p_{k+1} t \\
= & \Lambda_{k} p_{1 k} T+\lambda_{k+1} p_{k+1} t \\
= & p_{1 k} W-\left(p_{1 k}-p_{k+1}\right) \lambda_{k+1} t .
\end{aligned}
$$

Hence, combining (42) and (44), we have

$$
\begin{aligned}
0 \leq & V^{*}-V^{\mathrm{sw}} \\
\leq & -\left(p_{1 k}-p_{k+1}\right) \lambda_{k+1} t+\left(p_{1 k}-p_{1, k+1}\right) \mathrm{E}\left[W-N\left(\Lambda_{k}(T-t)\right)\right]^{+} \\
& +p_{1, k+1} \mathrm{E}\left[W-N\left(\Lambda_{k} T+\lambda_{k+1} t\right)\right]^{+} .
\end{aligned}
$$


Since

$$
p_{1 k}\left(\Lambda_{k+1}-\lambda_{k+1}\right)=\lambda_{1} p_{1}+\cdots+\lambda_{k} p_{k}=p_{1, k+1} \Lambda_{k+1}-p_{k+1} \lambda_{k+1},
$$

we can write

$$
\left(p_{1 k}-p_{k+1}\right) \lambda_{k+1} t=\left(p_{1 k}-p_{1, k+1}\right)\left[W-\Lambda_{k}(T-t)\right] .
$$

Furthermore,

$$
\begin{aligned}
\mathrm{E}\left[W-N\left(\Lambda_{k}(T-t)\right)\right]^{+} & =\mathrm{E}\left[W-\Lambda_{k}(T-t)+\Lambda_{k}(T-t)-N\left(\Lambda_{k}(T-t)\right)\right]^{+} \\
& \leq W-\Lambda_{k}(T-t)+\mathrm{E}\left[\Lambda_{k}(T-t)-N\left(\Lambda_{k}(T-t)\right)\right]^{+},
\end{aligned}
$$

where the inequality follows from $W \geq \Lambda_{k}(T-t)$. Hence, the first two terms on the right hand side of (46) can be combined to yield the following:

$$
\begin{aligned}
0 & \leq V^{*}-V^{\mathrm{sw}} \\
& \left.\leq\left(p_{1 k}-p_{1, k+1}\right) \mathrm{E}\left[\Lambda_{k}(T-t)-N\left(\Lambda_{k}(T-t)\right)\right]^{+}\right]+p_{1, k+1} \mathrm{E}[W-N(W)]^{+} .
\end{aligned}
$$

From (45), we know the two terms on the right side above are of order $O(\sqrt{T})$ and $O(\sqrt{W})$, respectively. On the other hand, $V^{*}$ is clearly of order $O(W)$ - both the upper and lower bounds in Lemmas 4 and 5 are of order $O(W)$. Hence, dividing both sides above by $V^{*}$ and letting $W \rightarrow \infty$ results in $V^{S W} / V^{*} \rightarrow 1$.

Next, consider the case of $c>\Lambda_{1}^{-1}$, which corresponds to the case of $\Lambda_{1} T>W$. From Lemmas 4 and 5, we have

$$
\begin{aligned}
0 \leq V^{*}-V^{\mathrm{sw}} & \leq p_{1} W-p_{1} \mathrm{E}[W \wedge N(W)]^{+} \\
& =p_{1} \mathrm{E}[W-N(W)]^{+} \sim O(\sqrt{W})
\end{aligned}
$$

In this case, $V^{*}$ is still of order $O(W)$; and hence, $V^{\mathrm{SW}} / V^{*} \rightarrow 1$. Finally, in the case of $c<\Lambda_{m}^{-1}$, which corresponds to $\Lambda_{m} T<W$, we have

$$
\begin{aligned}
0 \leq V^{*}-V^{\mathrm{sw}} & \leq p_{1 m} \Lambda_{m} T-p_{1 m} \mathrm{E}\left[W \wedge N\left(\Lambda_{m} T\right)\right]^{+} \\
& \leq p_{1 m} \Lambda_{m} T-p_{1 m} \mathrm{E}\left[\Lambda_{m} T \wedge N\left(\Lambda_{m} T\right)\right]^{+} \\
& =p_{1 m} \mathrm{E}\left[\Lambda_{m} T-N\left(\Lambda_{m} T\right)\right]^{+} \sim O(\sqrt{T}) .
\end{aligned}
$$

In this case, $V^{*}$ is of order $O(T)$; hence, $V^{\mathrm{SW}} / V^{*} \rightarrow 1$ follows when $T \rightarrow \infty$.

Example 2 We continue with the example in $\$ 4.3$, but suppose the batch size $Q$ follows negative binomial distributions:

$$
\mathrm{P}[Q=k]=\left(\begin{array}{c}
k+r-1 \\
r-1
\end{array}\right) p^{r}(1-p)^{k}, \quad k=0,1,2, \cdots
$$




\begin{tabular}{||c|c|c|cc|cc||}
\hline$W$ & $(r, p)$ & Optimal & Switch & \% off & Equal & $\%$ off \\
\hline 20 & $(4,0.33)$ & 17.59 & 17.47 & $0.67 \%$ & 17.34 & $1.37 \%$ \\
& $(8,0.5)$ & 17.68 & 17.50 & $0.99 \%$ & 17.28 & $2.27 \%$ \\
\hline 40 & $(4,0.33)$ & 34.39 & 34.16 & $0.67 \%$ & 33.99 & $1.18 \%$ \\
& $(8,0.5)$ & 34.60 & 34.26 & $0.97 \%$ & 34.14 & $1.34 \%$ \\
\hline 60 & $(4,0.33)$ & 50.08 & 49.62 & $0.92 \%$ & 48.74 & $2.69 \%$ \\
& $(8,0.5)$ & 50.39 & 49.84 & $1.09 \%$ & 49.14 & $2.48 \%$ \\
\hline \multirow{2}{*}{160} & $(4,0.33)$ & 101.43 & 100.87 & $0.55 \%$ & 72.85 & $28.18 \%$ \\
& $(8,0.5)$ & 102.38 & 101.84 & $0.52 \%$ & 72.72 & $28.97 \%$ \\
\hline 180 & $(4,0.33)$ & 104.54 & 104.37 & $0.16 \%$ & 72.69 & $30.47 \%$ \\
& $(8,0.5)$ & 105.48 & 105.31 & $0.16 \%$ & 73.14 & $30.66 \%$ \\
\hline 200 & $(4,0.33)$ & 105.74 & 105.69 & $0.05 \%$ & 72.73 & $31.20 \%$ \\
& $(8,0.5)$ & 105.78 & 105.76 & $0.01 \%$ & 72.56 & $30.82 \%$ \\
\hline \hline
\end{tabular}

Table 1: Performance of the policies as $W$ increases (with fixed $T$ ).

\begin{tabular}{||c|c|c|c|c||c|c|c|c|c||}
\hline$W, T$ & $(r, p)$ & Optimal & Switch & \% off & $W, T$ & $(r, p)$ & Optimal & Switch & \% off \\
\hline 20,20 & $(4,0.33)$ & 17.59 & 17.47 & $0.67 \%$ & 40,40 & $(4,0.33)$ & 36.74 & 36.55 & $0.52 \%$ \\
& $(8,0.5)$ & 17.68 & 17.50 & $0.99 \%$ & & $(8,0.5)$ & 36.87 & 36.60 & $0.73 \%$ \\
\hline 60,60 & $(4,0.33)$ & 55.62 & 55.38 & $0.43 \%$ & 80,80 & $(4,0.33)$ & 75.11 & 74.95 & $0.21 \%$ \\
& $(8,0.5)$ & 55.71 & 55.41 & $0.54 \%$ & & $(8,0.5)$ & 75.14 & 74.97 & $0.23 \%$ \\
\hline
\end{tabular}

Table 2: Performance of the switch-over policy with simultaneous increase of both $W$ and $T$.

and hence,

$$
\mathrm{E}[Q]=\frac{r(1-p)}{p}, \quad \operatorname{Var}[Q]=\frac{r(1-p)}{p^{2}} .
$$

We choose $(r, p)$ to be $(4,0.33)$ and $(8,0.5)$. While both distributions have the same mean 8 , their coefficients of variation are 3 and 2 , respectively.

In Table 1, we compare the performance between the optimal policy and the switch-over policy, with the switch-over times optimized (the column under "Switch"). Fixing $T=20$, we increase the value of $W$. The relative error between the two policies first has a slight increase, and then quickly decreases as $W$ becomes large (8-10 times the value of $T$ ). In addition, we list in the table the performance of a switch-over policy with equally spaced switch-over times (the column under "Equal"), i.e., $y_{i}=T / 4=5$ for all $i$. The results indicate that the performance of this policy deteriorates rather severely as $W$ increases. That is, it is crucial to optimize the switch-over times.

Next, in Table 2, we increase $T$ simultaneously with $W$. The relative error of the switch-over policy appears to decrease monotonically. 


\section{Pricing Models}

As mentioned in the introductory section, an important motivation for us to study the switchover policy is to use it as a means to solve optimal pricing problems. (In this sense, the prices derived below are only optimal with respect to the switch-over policy.) Suppose, instead of assuming all prices are given, and ordered as in (7), it is now our decision to come up with the $m-1$ "discount" prices, $p_{2} \geq \cdots \geq p_{m}$. The original price $p_{1}$ is still given, and $p_{1} \geq p_{2}$. (The case when $p_{1}$ is also a decision variable is discussed at the end of this section.)

Here, $m$ is also assumed to be given. That is, we limit the number of price takedowns that can take place over the horizon. Furthermore, we assume the time horizon is divided into $m$ equal segments, with one price for each segment. Without loss of generality, assume $T=m$, hence each segment is of unit length. Note that the equal-length segments do not contradict the earlier models, where the time lengths $y_{\ell}$ corresponding to accommodating different prices are decision variables, and hence may vary in $\ell$. It can happen that in the derived pricing solution we have $p_{\ell}=p_{\ell+1}$, for instance, then the price $p_{\ell}$ will apply to two time segments instead of one.

Consider the model in \$4.1, i.e., batch Poisson order arrivals, with the batch size independent of the price (hence, we are not modeling phenomena such as quantity discount); however, let the arrival rate be a decreasing function of the price, $\lambda(p)$, which is also assumed to be differentiable.

Following the analysis that leads to the problem formulation in (37), and noticing that $p_{1 i}$ becomes $p_{i}$ here, since now there is only a single price in each time segment, we have the following optimization problem:

$$
\begin{aligned}
& \max \quad p_{1}\left[W-G\left(\mu_{1}\right)\right]+p_{2}\left[G\left(\mu_{1}\right)-G\left(\mu_{2}\right)\right]+\cdots+p_{m}\left[G\left(\mu_{m-1}\right)-G\left(\mu_{m}\right)\right] \\
& =p_{1} W_{1}-\left(p_{1}-p_{2}\right) G\left(\mu_{1}\right)-\cdots-\left(p_{m-1}-p_{m}\right) G\left(\mu_{m-1}\right)-p_{m} G\left(\mu_{m}\right) \text {, }
\end{aligned}
$$

where $\left(p_{2}, \ldots, p_{m}\right)$ are decision variables, satisfying the ordering in (7); and

$$
\mu_{i}=\Lambda_{i}=\lambda\left(p_{1}\right)+\cdots+\lambda\left(p_{i}\right), \quad i=1, \ldots, m
$$

Equivalently, we can solve the following minimization problem:

$$
\begin{aligned}
\min _{p_{2}, \ldots, p_{m}} & \left(p_{1}-p_{2}\right) G\left(\mu_{1}\right)+\cdots+\left(p_{m-1}-p_{m}\right) G\left(\mu_{m-1}\right)+p_{m} G\left(\mu_{m}\right), \\
\text { s.t. } & p_{1} \geq p_{2} \geq \cdots \geq p_{m} \geq 0 .
\end{aligned}
$$

The above problem, although no longer separable, can still be solved by a standard nonlinear (convex) programming algorithm. Below, we present an approximation algorithm that is easy to run and appears to work quite well. 
Apply a transformation of variable: let the new decision variables be

$$
r_{m}:=p_{m} ; \quad r_{i}:=p_{i}-p_{i+1}, \quad i=1, \ldots, m-1 .
$$

The optimization problem in (48) then becomes as follows:

$$
\min _{r} \sum_{i=1}^{m} r_{i} G\left(\mu_{i}\right), \quad \text { s.t. } \quad r_{1}+\cdots+r_{m}=p_{1} ; \quad r_{i} \geq 0, \quad i=1, \ldots, m .
$$

Write the derivatives

$$
\lambda_{i}^{\prime}:=\lambda^{\prime}\left(p_{i}\right), \quad \Lambda_{i}^{\prime}:=\lambda_{1}^{\prime}+\cdots+\lambda_{i}^{\prime}
$$

We can derive, for any $i$ and $j$,

$$
\frac{\partial \mu_{i}}{\partial r_{j}}=\Lambda_{i \wedge j}^{\prime}
$$

This follows from noticing that when $i>j, p_{i}=r_{i}+\cdots+r_{m}$ does not involve $r_{j}$.

Therefore, the optimality equations are:

$$
G\left(\mu_{j}\right)+\sum_{i=1}^{m} r_{i} G^{\prime}\left(\mu_{i}\right) \Lambda_{i \wedge j}^{\prime}=\eta, \quad j=1, \ldots, m .
$$

Taking the difference between two consecutive equations above, we have

$$
G\left(\mu_{j}\right)-G\left(\mu_{j-1}\right)+\lambda_{j}^{\prime} \sum_{i=j}^{m} r_{i} G^{\prime}\left(\mu_{i}\right)=0, \quad j=2, \ldots, m
$$

From the above, we can derive:

$$
r_{j}=\frac{G\left(\mu_{j-1}\right)-G\left(\mu_{j}\right)}{\lambda_{j}^{\prime} G^{\prime}\left(\mu_{j}\right)}-\frac{1}{G^{\prime}\left(\mu_{j}\right)} \sum_{i=j+1}^{m} r_{i} G^{\prime}\left(\mu_{i}\right) \quad j=2, \ldots, m .
$$

Now, ignoring the second term on the right hand side above, we have the following approximation:

$$
r_{j}=\frac{G\left(\mu_{j-1}\right)-G\left(\mu_{j}\right)}{\lambda_{j}^{\prime} G^{\prime}\left(\mu_{j}\right)}, \quad j=2, \ldots, m
$$

Observe that

$$
\lambda_{j}=\lambda\left(r_{j}+\cdots+r_{m}\right)=\lambda\left(p_{1}-r_{1}-\cdots-r_{j-1}\right)
$$

only involves $\left(r_{1}, \ldots, r_{j-1}\right)$; and hence, so does $\mu_{j}$. That is, the right hand side of (53), for each $j$, only involves $\left(r_{1}, \ldots, r_{j-1}\right)$. Therefore, following the recursion, we can relate $r_{2}, \ldots, r_{m}$ all to $r_{1}$, and then derive $r_{1}$ through a simple line search via the equation

$$
r_{1}+r_{2}+\cdots+r_{m}=p_{1}
$$




\begin{tabular}{||c|c|cccccccc|c||}
\hline$(W ; a, b)$ & $i=$ & 1 & 2 & 3 & 4 & 5 & 6 & 7 & 8 & obj. val. \\
\hline$(40 ; 15,14)$ & $p_{i}$ & 1 & 0.69 & 0.69 & 0.69 & 0.69 & 0.69 & 0.68 & 0.62 & 25.64 \\
$(40 ; 40,37.33)$ & $p_{i}$ & 1 & 0.63 & 0.61 & & & & & & 22.94 \\
\hline$(40 ; 15,2)$ & $p_{i}$ & 1 & 0.60 & 0.60 & 0.60 & 0.60 & 0.60 & 0.59 & 0.56 & 20.77 \\
$(40 ; 40,2)$ & $p_{i}$ & 1 & 0.55 & 0.54 & & & & & & 19.95 \\
\hline$(40 ; 2,1.5)$ & $p_{i}$ & 1 & 0.57 & 0.57 & 0.57 & 0.57 & 0.57 & 0.54 & 0.37 & 20.46 \\
$(40 ; 5.33,1.5)$ & $p_{i}$ & 1 & 0.52 & 0.44 & & & & & & 20.10 \\
\hline
\end{tabular}

Table 3: Optimal pricing under linear, exponential and power demand functions.

In executing this recursion, we have noticed that when $p_{1}$ is not large enough, $r_{1}$ might become negative while we solve the equation in (54). This is because the other $r_{j}$ 's, $j \neq 1$, are all positive, as evident from (53). One way to avoid this from happening is to first replace $p_{1}$ by $C p_{1}$, where $C$ is a large positive number. Once all the $r_{j}$ 's are derived and $r_{1}$ is positive, divide each of these by $C$.

In the following examples, we consider three commonly used functions, the linear, exponential and power functions, that model the relation between the arrival rate and the price:

$$
\lambda(p)=a-b p, \quad \lambda(p)=a e^{-b p}, \quad \lambda(p)=\frac{a}{p^{b}} ;
$$

where $a$ and $b$ are positive parameters in all three cases. The corresponding derivatives are:

$$
\lambda^{\prime}(p)=-b, \quad \lambda^{\prime}(p)=-b \lambda(p), \quad \lambda^{\prime}(p)=-\frac{b}{p} \lambda(p) .
$$

Example 3 In this example we choose $p_{1}=1$, and consider the linear, exponential and power functions in (55) with different parameters $(a, b)$, along with different $W$ values. The choice of the parameters $(a, b)$ is such that all three functions coincide at $p=1$; the linear and exponential functions also coincide at $p=0$; while the power function coincides with the linear function at a point close to the origin.

We list both the optimal solutions and the approximations following (53). In each case, we consider both $m=8$ and $m=3$. The results are summarized in Table 3, where listed under "obj. val." are the original, maximal objective values following (47) (in particular, including the $p_{1} W$ term).

Example 4 Next, we examine the optimal price reduction with respect to the available inventory $W$. We take the exponential demand case above, fix $m=8$ and $(a, b)=(15,2)$, while changing $W$. The results are displayed in Table 4. 


\begin{tabular}{||c|c|cccccccc|c||}
\hline$W$ & $i=$ & 1 & 2 & 3 & 4 & 5 & 6 & 7 & 8 & obj. val. \\
\hline 50 & $p_{i}$ & 1 & 0.52 & 0.52 & 0.52 & 0.52 & 0.52 & 0.52 & 0.52 & 21.28 \\
\hline 30 & $p_{i}$ & 1 & 0.73 & 0.73 & 0.73 & 0.73 & 0.73 & 0.71 & 0.61 & 19.35 \\
\hline 25 & $p_{i}$ & 1 & 0.82 & 0.82 & 0.82 & 0.82 & 0.81 & 0.78 & 0.65 & 18.12 \\
\hline 20 & $p_{i}$ & 1 & 0.93 & 0.93 & 0.93 & 0.93 & 0.92 & 0.86 & 0.69 & 16.43 \\
\hline 15 & $p_{i}$ & 1 & 1 & 1 & 1 & 1 & 1 & 1 & 0.78 & 14.00 \\
\hline 10 & $p_{i}$ & 1 & 1 & 1 & 1 & 1 & 1 & 1 & 0.88 & 9.90 \\
\hline
\end{tabular}

Table 4: Optimal pricing under various inventory levels.

\begin{tabular}{||c|c|ccc|c|c||}
\hline$(W ; a, b)$ & $i=$ & 1 & 2 & 3 & obj. val. & $\%$ off opt \\
\hline$(40 ; 40,37.33)$ & $p_{i}$ & 1 & 0.66 & 0.45 & 21.82 (approx) & $5 \%$ \\
& $p_{i}$ & 1 & 1 & 1 & 8.00 (non-opt) & $65 \%$ \\
\hline$(40 ; 40,2)$ & $p_{i}$ & 1 & 0.68 & 0.35 & 19.10 (approx) & $4 \%$ \\
& $p_{i}$ & 1 & 0.90 & 0.90 & 17.32 (non-opt) & $13 \%$ \\
\hline$(40 ; 5.33,1.5)$ & $p_{i}$ & 1 & 0.68 & 0.36 & 19.84 (approx) & $1 \%$ \\
& $p_{i}$ & 1 & 1 & 0.50 & 18.21 (non-opt) & $10 \%$ \\
\hline
\end{tabular}

Table 5: Approximations and non-optimal pricing schemes.

Example 5 Finally, we examine the approximation scheme in (53), which solves the optimality equations approximately. We take the above cases under $m=3$. We also examine non-optimal alternatives that offer different levels of discount at different periods or no discount at all. (For instance, in the second case, $(1,0.90,0.90)$ indicates full price in period 1 , and $10 \%$ discount in the remaining two periods.) The results are summarized in Table 5, where the last column is the percentage off the objective value under optimal pricing.

From the above results, we observe that

(a) reducing the number of price markdowns from $7(m=8)$ to $2(m=3)$ has a rather minor effect on the objective values;

(b) with more inventory available for sale, price reduction becomes more substantial and starts earlier, as expected;

(c) the approximation scheme in (53) performs quite well in all three cases;

(d) applying the optimal pricing results in a substantial advantage over other ad-hoc schemes.

The model and analysis discussed above extend readily to the case where, in addition to the discount prices, the original price $p_{1}$ is also a decision variable. The optimization problem 
now becomes

$$
\begin{array}{ll}
\max _{p_{1}, r} & p_{1} W-\sum_{i=1}^{m} r_{i} G\left(\mu_{i}\right) \\
\text { s.t. } & r_{1}+\cdots+r_{m} \geq p_{1} ; \quad p_{1} \geq 0 ; r_{i} \geq 0, i=1, \ldots, m .
\end{array}
$$

The Lagrangian is

$$
p_{1} W-\sum_{i=1}^{m} r_{i} G\left(\mu_{i}\right)+\eta\left(r_{1}+\cdots+r_{m}-p_{1}\right),
$$

with $\eta$ being the multiplier. Therefore, the optimality equations in (52) still apply; and in addition, we have $\eta=W$ (from setting the partial derivative with respect to $p_{1}$ to zero). Similarly, the recursion in (53) also applies, assuming $p_{1}$, as well as $r_{1}$, is given; and $r_{1}$ is still obtained from the summation constraint: $r_{1}+\cdots+r_{m}=p_{1}$. (That this constraint must be binding follows from the fact that the term, $\sum_{i=1}^{m} r_{i} G\left(\mu_{i}\right)$, is increasing in $\left(r_{1}, \ldots, r_{m}\right)$, which can be directly verified.) Finally, $p_{1}$ can be obtained from another line search to enforce $\eta=W$.

\section{Concluding Remarks}

The models discussed here can be extended in a number of ways. First, the demand function in the pricing model as represented by the Poisson arrival rate can depend on time (period), $i$, as well as on price $p_{i}$, to take the form $\lambda\left(i, p_{i}\right)$. For instance, a discount that takes place earlier might attract more (or, less) demand. The results in $\$ 6$ will continue to hold, since the only change needed is from $\mu_{i}=\lambda\left(p_{i}\right)$ to $\mu_{i}=\lambda\left(i, p_{i}\right)$.

Second, the available inventory $W$ can be made a decision variable too. This amounts to constructing a newsvendor problem on top of the switch-over or pricing models developed here. It is readily verified that all the objective functions (for maximization) involved are concave in $W$. Hence, with the addition of replenishment and salvage costs, the resulting newsvendor model can be routinely solved, once the optimal switching points or optimal prices are obtained (for each given $W$ ).

Third, in the switch-over policy, we can incorporate a service measure such as the acceptance rate for each price class $k$ : recall, it will not be accepted until the time interval $\left(t_{k-1}, t_{k}\right]$. Consider the model in 4.1, for instance. We know the total expected number of accepted units, over the time interval $\left(t_{k-1}, t_{k}\right]$ is $G\left(\mu_{k-1}\right)-G\left(\mu_{k}\right)$, of which the share of class $k$ is proportion to $\lambda_{k} / \Lambda_{k}$. Hence, the expected number of accepted units for class $k$ over the entire horizon $(0, T]$ is:

$$
\sum_{\ell=k}^{m}\left[G\left(\mu_{k-1}\right)-G\left(\mu_{k}\right)\right] \frac{\lambda_{k}}{\Lambda_{\ell}}
$$


The above divided by the expected number of class $k$ arrivals over the horizon, $\lambda_{k} T \mathrm{E}(Q)$, is what we call average acceptance rate for class $k$, denoted $\alpha_{k}$. We can hence derive $\alpha_{k}$ after the optimal switch-over points are derived; or, include a minimal requirement for $\alpha_{k}$ as a constraint.

\section{References}

[1] Bitran, G. And Mondschein, S., An Application of Yield Manangement to the Hotel Industry Considering Multiple Day Stays. Operations Research, 43 (1995), 427-443.

[2] Brummelle, S. and Walczak, D., Dynamic Airline Revenue Management with Multiple Semi-Markov Demand. Operations Research, 51 (2003), 137-148.

[3] Cooper, W. Asymptotic Behavior of an Allocation Policy for Revenue Management, Operations Research, 50, 720-727.

[4] Bicheler, M., Et Al., Applications of Flexible Pricing in Business-to-Business Electronics Commerce. IBM System Journal, (April, 2002).

[5] CaO, H., Jang, Y., And Lu, Y., Bid Pricing with Incomplete Data via an EM Approach. Working paper, IBM T.J. Watson Research Center, 2002.

[6] Feng, Y. And Gallego, G., Optimal Stopping Times for End of Seasons Sales and Optimal Stopping Times for Promotional Fares. Management Science, 41 (1995), 13721391.

[7] Feng, Y. and Gallego, G., Perishable Asset Revenue Management with Markovian Time Dependent Demand Intensities. Management Science, 46 (2000), 941-956.

[8] Feng, Y. And Xiao, B., Optimal Policies of Yield Management with Multiple Predetermined Prices. Operations Research, 48 (2000), 332-343.

[9] Gallego, G. and van Ryzin, G., A multiproduct dynamic pricing problem and its applications to network yield management, Operations Research, 45 (1997), 24-41.

[10] Kleywegt, A.J. and Papastavrou, J.D., The Dynamic and Stochastic Knapsack Problem. Operations Research, 46 (1998), 17-35.

[11] Kleywegt, A.J. and Papastavrou, J.D., The Dynamic and Stochastic Knapsack Problem with Random Sized Items. Operations Research 49 (2001), 26-41.

[12] Lee, T.C. And Hersh, M., A Model for Airline Seat Inventory Control with Multiple Seat Booking. Transpotation Sci. 27 (1993), 1252-1265.

[13] Lippman, S., Applying a New Device in the Optimization of Exponential Queueing Systems, Operations Research, 23 (1975), 687-710

[14] Maglaras, C. and Zeevi, A., Pricing and Design of Differentiated Services: Approximate Analysis and Structural Insights. Operations Research, 53 (2005), 242-262. 
[15] Papastavrou, J.D., Rajagopalan, S., and Kleywegt, A.J., The Dynamic and Stochastic Knapsack Problem with Deadlines. Management Science, 42 (1996), 1706-1718.

[16] Ross, K.W. and Tsang, D., The Stochastic Knapsack Problem. IEEE Transactions on Communications, 34 (1989), 47-53.

[17] Ross, K.W. and Yao, D.D., Monotonicity Properties of the Stochastic Knapsack. IEEE Transactions on Information Theory, 36 (1990), 1173-1179.

[18] Shanthikumar, J.G. And Yao, D.D., Strong Stochastic Convexity: Closure Properties and Applications. Journal of Applied Probability, 28 (1991), 131-145.

[19] Talluri, K. And van Ryzin, G., A randomized linear programming method for computing network bid prices, Transportation Science 33, 1999, 207-216.

[20] Van Slyke, R. and Young, Y., Finite Horizon Stochastic Knapsacks with Applications in Yield Management. Operations Research, 48 (2000), 155-172.

[21] Zhao, W. And Zheng, Y., Optimal Dynamic Pricing for Perishable Assets with Nonhomogeneous Demand. Management Science, 46 (2000), 375-388. 